\title{
The novel early predictive marker presepsin for postoperative pancreatic fistula: A pilot study
}

\author{
MASATSUGU HIRAKI $^{1}$, ATSUSHI MIYOSHI ${ }^{1}$, EIJI SADASHIMA ${ }^{2}$, YUKIO SHINKAI $^{3}$, \\ MICHIO YASUNAMI $^{3}$, TATSUYA MANABE ${ }^{4}$, KENJI KITAHARA ${ }^{1}$ and HIROKAZU NOSHIRO ${ }^{4}$ \\ ${ }^{1}$ Department of Surgery; ${ }^{2}$ Life Science Research Institution; ${ }^{3}$ Clinical Laboratory, Saga Medical Center Koseikan, \\ Saga 840-8571; ${ }^{4}$ Department of Surgery, Saga University Faculty of Medicine, Saga 849-8501, Japan
}

Received January 3, 2020; Accepted March 26, 2020

DOI: $10.3892 / \mathrm{etm} .2020 .8919$

\begin{abstract}
Postoperative pancreatic fistula (PF) is a major and serious complication that occurs after pancreaticoduodenectomy (PD). The aim of the current study was to evaluate the use of a novel biomarker, presepsin, for predicting clinically relevant postoperative pancreatic fistula (CR-POPF) after PD. A prospective pilot study was conducted using 30 consecutive patients who underwent PD. Risk factors and candidates for predictive biomarkers for CR-POPF were statistically analyzed. CR-POPF (grade B and C; determined according to the guidelines of the International Study Group of Pancreatic Fistula) occurred in 15 patients $(50 \%)$. Univariate analysis revealed that certain underlying conditions, including non-pancreatic cancer, smaller pancreatic ducts and soft pancreas texture were significantly associated with CR-POPF $(\mathrm{P}=0.005$, $\mathrm{P}=0.004$ and $\mathrm{P}=0.014$, respectively). Furthermore, on day 1 post surgery ( $\mathrm{POD} 1)$, white blood cell count $(\mathrm{P}=0.040)$, levels of serum amylase $(\mathrm{P}=0.002)$ and serum presepsin $(\mathrm{P}=0.012)$, and the concentration of presepsin in drainage fluid $(\mathrm{P}<0.001)$ were significantly increased in CR-POPF compared with non-CR-POPF cases. Receiver operating characteristic curve analyses revealed that, on POD1, serum amylase and the concentration of presepsin in drainage fluid had an area under the curve value exceeding 0.8. A multivariate logistic regression analysis revealed that a higher concentration of presepsin in the drainage fluid was an independent predictive marker for CR-POPF (odds ratio, 14.503; 95\% confidence interval, 1.750-120.229; $\mathrm{P}=0.013$ ). To the best of our knowledge, the present study demonstrated for the first time that presepsin concentration in drainage fluid is a useful marker of CR-POPF after PD.
\end{abstract}

Correspondence to: Dr Masatsugu Hiraki, Department of Surgery, Saga Medical Center Koseikan, 400 Nakabaru, Kasemachi, Saga 840-8571, Japan

E-mail: masatsuguhiraki@hotmail.com

Key words: presepsin, sCD14-ST, soluble CD14 subtype, pancreatic fistula, pancreaticoduodenectomy

\section{Introduction}

Pancreaticoduodenectomy (PD) is an operative method for treating patients with pancreatic, biliary tract or duodenal neoplasm. However, this surgical procedure is strongly associated with perioperative high morbidity and mortality rates, ranging from 35-60 and 0-4\%, respectively (1-6). Predominant serious postoperative complications after PD involve pancreatic fistula (PF), intraperitoneal bleeding, intra-abdominal abscess, delayed gastric emptying and bile leakage. Of these, $\mathrm{PF}$ is observed particularly frequently after PD, consequently being associated with prolonged hospitalization, increased medical costs and delayed introduction of postoperative chemotherapy, resulting in an increased mortality rate if bacterial infection, intra-abdominal abscess or bleeding follow PF. As the incidence of clinically relevant postoperative pancreatic fistula (CR-POPF) after PD reaches approximately $12-30 \%(2,4-10)$, the early detection of this condition is very important for managing patients who have undergone PD.

Presepsin has been used as a novel biomarker for detecting bacterial infection $(11,12)$. Presepsin is also known as soluble CD14 subtype that is released into the circulation after the activation of the pro-inflammatory signal cascade on contact with infectious agents $(12,13)$. Previous studies have shown that serum presepsin is useful for the early detection of bacterial infection and sepsis $(11,12)$. POPF is considered to be closely associated with bacterial infection $(8,14)$. Indeed, surgical site bacterial infection involves the secretion of certain protease activators in the pancreatic juice that convert trypsinogen to trypsin, subsequently inducing CR-POPF (14). However, no study has focused on the utility of presepsin as a predictive marker for CR-POPF.

We hypothesized that presepsin might be a useful biomarker for the early detection of CR-POPF after PD.

\section{Materials and methods}

Patients. A prospective pilot study was conducted in a single institute (Department of Surgery, Saga Medical Center Koseikan) for 30 consecutive patients who underwent PD for various primary diseases between May 2017 and March 2019. All patients and their families were fully informed about the surgical procedures in order to obtain their written consent, including broad consent to participate in this study. The medical 
ethics committee of Saga Medical Center Koseikan reviewed and approved this study design (permission nos. 17-01-01-04, 19-05-01-01).

The primary diseases of the pancreatic, biliary tract or duodenal neoplasms were diagnosed preoperatively by various imaging modalities and/or a pathological examination. Biliary stent drainage was performed during endoscopic retrograde cholangiopancreatography for patients manifesting obstructive jaundice.

Surgical procedure and postoperative management. All operations were performed by experienced surgeons whose quality was certified by the Japanese Society of Hepato-Biliary-Pancreatic Surgery or supervised by such expert surgeons. All patients were subjected to the same surgical procedure as follows: Patients received subtotal stomach-preserving PD with regional lymph node dissection according to the presence of malignant disease or potential malignancy. The digestive tract was reconstructed by the modified Child's method with ante-colic gastro-jejunostomy. Pancreatico-jejunostomy was performed by duct-to-mucosa anastomosis in addition to pull-through adhesive anastomosis using the modified Blumgard procedure (15). Duct-to-mucosa anastomosis was performed with double-layer anastomosis using 3-0 non-absorbable monofilament sutures and 4-0 absorbable sutures. A 4- to 6-Fr polyvinyl chloride tube in the pancreatic duct and 3-mm polyvinyl chloride tube in the hepatic bile duct were inserted individually for external drainage. In addition, a couple of drainage tubes were placed around the pancreaticojejunostomy and hepaticojejunostomy for intra-abdominal drainage. The drainage fluid was continuously suctioned and collected for a later analysis. Fresh sample of the drainage fluid were collected in the morning and analyzed. Tube gastrostomy was not used in any case.

All of the patients received an intravenous drip infusion of antibiotics with $1 \mathrm{~g}$ of cefmetazole sodium every $3 \mathrm{~h}$ during the operation and $1 \mathrm{~g}$ of cefmetazole sodium twice a day on postoperative day (POD) 1 and 2. The intra-abdominal drainage tubes were removed or gradually pull out when CR-POPF and intra-abdominal infection were deemed negligible. It is considered as below: Inflammatory markers are improved and/or the volume of the drainage fluid is reduced without bacterial detection. After the removal of the intra-abdominal drainage tubes, the external stent tubes in the pancreatic duct and hepatic bile duct were removed.

Statistical analyses. The following data were collected from the prospectively maintained comprehensive database or medical records: Gender, age, body mass index, American Society of Anesthesiologists (ASA) physical status score, history of diabetes mellitus, current smoking habit, prior abdominal operations, presence of preoperative biliary drainage, diameter of the main pancreatic duct, origin of the primary disease, TNM-staging, presence of extended vascular resection, operative time, intra- and post-operative blood transfusion, pancreatic texture, presence of postoperative complications, length until removal of the surgical drainage tube, length of the post-operative hospital stay and pre- and post-operative laboratory data.

PF was graded according to the guideline of the International Study Group on PF (ISGPF) in 2016 (16),
Table I. Patients characteristics.

\begin{tabular}{lc}
\hline Characteristic & $\begin{array}{c}\text { Patients } \\
(\mathrm{n}=30)\end{array}$ \\
\hline Sex (males:females) & $13: 17$ \\
Age [years, median $(\mathrm{min}$, max)] & $72(37,84)$ \\
Body mass index, $\mathrm{kg} / \mathrm{m}^{2}$; median & $22.6(18.7,30.7)$ \\
(min, max) & \\
ASA (PS1,2: PS3,4) & $27: 3$ \\
Diabetes mellitus (yes:no) & $9: 21$ \\
Currently smoking (yes:no) & $4: 26$ \\
Prior abdominal surgery (yes:no) & $3: 27$ \\
Preoperative biliary drainage (yes:no) & $17: 13$ \\
White blood cells, $\mu 1$; median & $5,300(3,300,9,700)$
\end{tabular}

(min, max)

Hemoglobin, g/dl; median (min, max)

$13.0(9.7,15.6)$

Albumin, g/dl; median (min, max)

Total bilirubin, $\mathrm{mg} / \mathrm{dl}$; median

$4.1(3.1,5.0)$

$0.9(0.3,2.7)$

(min, max)

C-reactive protein, $\mathrm{mg} / \mathrm{dl}$; median

(min, max)

Tumor kind (benign:malignant)

$0.08(0.01,1.28)$

Pancreatic cancer

$10(33.3 \%)$

Bile duct cancer

$10(33.3 \%)$

$7(23.3 \%)$

$3(10.0 \%)$

$15: 15$

Clinically relevant pancreatic

$10(33.3 \%)$

No amylase detected

$5(16.7 \%)$

Biochemical leak

$15(50.0 \%)$

Grade B

$0(0 \%)$

Grade C

$0: 30$

$6: 24$

$0: 30$

$0: 30$

$0: 30$

$0: 30$

$0: 30$

$0: 30$

$28(19,61)$

Post-operative hospital stay, days;

median (min, max)

ASA, American Society of Anesthesiologists.

and grades $\mathrm{B}$ and $\mathrm{C}$ were defined as indicating the presence of CR-POPF in this study. In brief, biochemical leak (BL) is clinically unimportant and is not referred to a true PF. A PF grade B requires a change in the postoperative management, namely the drainage tubes are either left in place $>3$ weeks or repositioned through endoscopic or percutaneous procedures. A PF Grade $\mathrm{C}$ requires reoperation since it can lead to organ failure and/or mortality as a result of the $\mathrm{PF}$. 
Table II. Univariate analysis of patients.

Clinically relevant postoperative pancreatic fistula

Characteristic

$(-) n=15$

(+) $n=15$

P-value

A, Patient characteristic

Sex (male:female)

Age, years; median (min, max)

Body mass index, $\mathrm{kg} / \mathrm{m}^{2}$; median (min, max)

ASA (PS1,2: PS3,4)

Diabetes mellitus (yes:no)

Current Smoking (yes:no)

Prior abdominal surgery (yes:no)

Preoperative biliary drainage (yes:no)

$5: 10$
$74(37,84)$
$22.4(18.7,27.3)$
$12: 3$
$5: 10$
$0: 15$
$2: 13$
$6: 9$

$\begin{array}{cr}8: 7 & 0.462 \\ 72(54,82) & 0.663 \\ 23.5(19.6,30.7) & 0.198 \\ 15: 0 & 0.224 \\ 4: 11 & >0.999 \\ 4: 11 & 0.100 \\ 1: 14 & >0.999 \\ 11: 4 & 0.139\end{array}$

$\mathrm{B}$, Preoperative laboratory data

White blood cells, $\mu \mathrm{l}$; median (min, $\max$ )

Hemoglobin, g/dl; median (min, $\max )$ )

Albumin, g/dl; median (min, $\max$ )

Total bilirubin, mg/dl; median (min, max)

C-reactive protein, $\mathrm{mg} / \mathrm{dl}$; median (min, $\max$ )

Tumor kind (benign:malignant)

Pancreatic cancer (yes:no)

Diameter of pancreatic duct, mm; median (min, max)

Tumor invasion (tumor limited to organ/tumor extends beyond organ)

Lymph node metastasis (negative:positive)

Distant organ metastasis (negative:positive)

Vascular resection (yes:no)

Operative time, min; median ( $\min , \max )$

Intraoperative bleeding, $\mathrm{ml}$; median (min, $\max$ )

Pancreas texture (hard:soft)

Intraoperative blood transfusion (yes:no)

Postoperative blood transfusion (yes:no)

$$
\begin{gathered}
4,700(3,500,8,600) \\
12.7(9.7,14.9) \\
4.1(3.2,5.0) \\
0.8(0.3,2.3) \\
0.06(0.02,1.28) \\
1: 14 \\
9: 6
\end{gathered}
$$

$5.49(1.43,12.36)$

$6: 9$

$8: 7$

$15: 0$

4: 11

$319(201,513)$

$427(71,1,100)$

$8: 7$

$1: 14$

$0: 15$

$\begin{array}{cr}6,200(3,300,9,700) & 0.237 \\ 13.0(10.4,15.6) & 0.281 \\ 4.0(3.1,4.7) & 0.348 \\ 1.2(0.4,2.7) & 0.140 \\ 0.10(0.01,1.04) & 0.775 \\ 2: 13 & >0.999 \\ 1: 14 & 0.005 \\ 1.83(1.15,4.42) & 0.004 \\ 7: 8 & >0.999 \\ 10: 5 & 0.710 \\ 14: 1 & >0.999 \\ 1: 14 & 0.330 \\ 358(217,701) & 0.071 \\ 400(170,1,194) & 0.740 \\ 1: 14 & 0.014 \\ 2: 13 & >0.999 \\ 1: 14 & >0.999\end{array}$

C, Postoperative laboratory data collected on POD1

Bacteria detected in the fluid collection (yes:no)

White blood cells, $\mu \mathrm{l}$; median (min, max)

C-reactive protein, $\mathrm{mg} / \mathrm{dl}$; median (min, $\max$ )

Serum amylase, IU/l; median (min, max)

Serum presepsin, pg/ml; median (min, max)

Presepsin in drainage fluid, pg/ml; median (min, max)

Post-operative hospital stay, days; median (min, max)

$1: 14$
$8,900(5,550,13,800)$
$8.33(5.27,14.08)$
$110(57,913)$
$219(141,801)$
$653(248,1,609)$
$25(22,48)$

0.598

0.040

0.772

0.002

0.012

$<0.001$

0.004

ASA, American Society of Anesthesiologists; POD1, post-operative day 1.

The summary data for continuous variables were expressed as the median and range (minimum, maximum). In the univariate analysis, patient subgroups were compared in terms of continuous variables by Wilcoxon's rank sum test for continuous variables and Fisher's exact test for binary variables. Receiver operation characteristics (ROC) analyses and the calculation of the area under the curve (AUC) were used to examine the capability of markers to diagnose PF. Continuous variables were converted into binary variables based on the optimal cut-off values using the ROC analyses. A multiple logistic regression analysis was performed to identify significant independent markers using two markers which showed a 

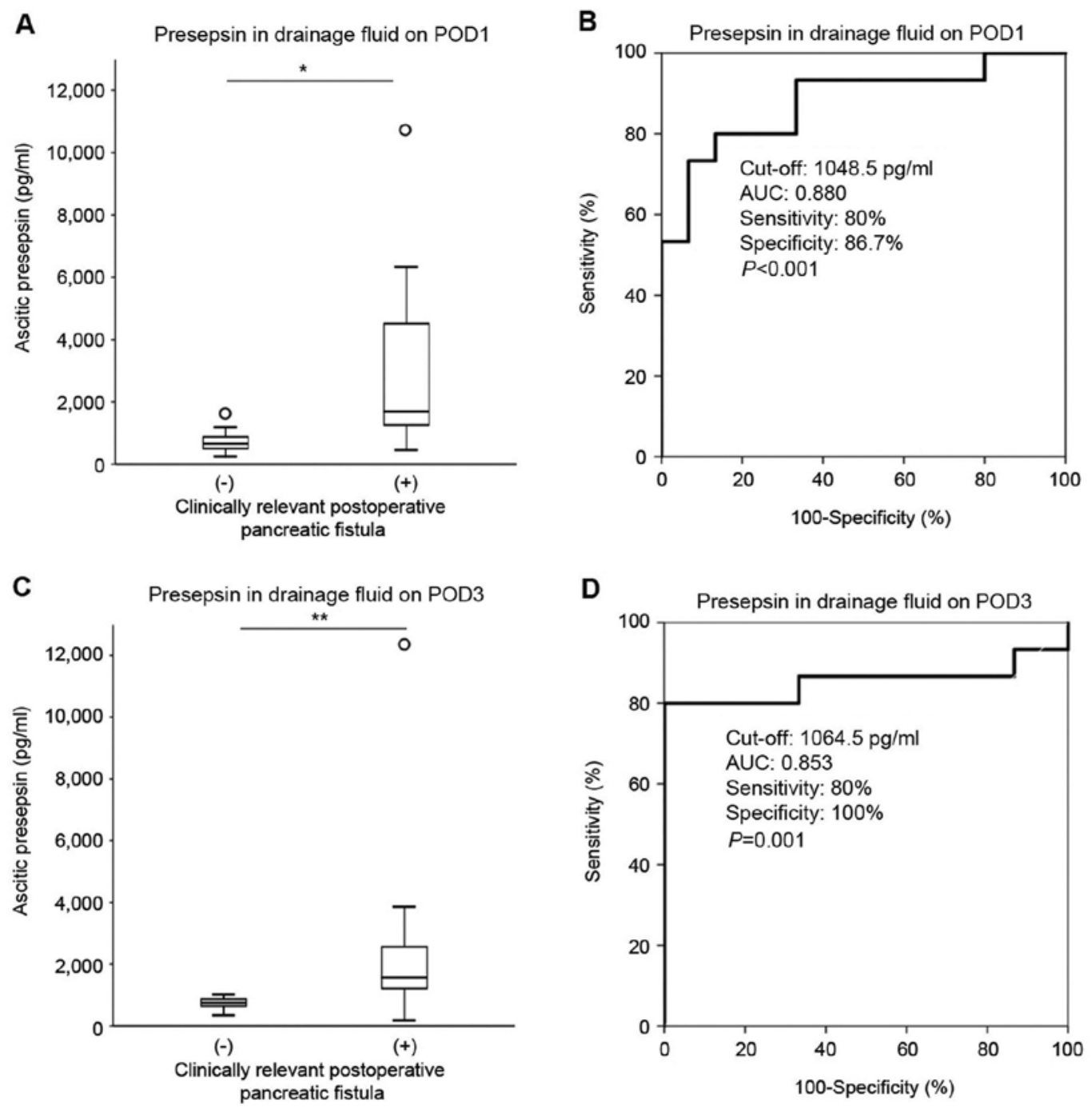

Figure 1. Ascitic presepsin. Presepsin levels in drainage fluid on (A) POD1 and (C) POD3 in patients without and with clinically relevant postoperative pancreatic fistulas. Receiver operative characteristic curves of presepsin levels in the drainage fluid of patients on (B) POD1 and (D) POD3. ${ }^{*} \mathrm{P}<0.001$ and ${ }^{* *} \mathrm{P}=0.001$. POD, post-operative day.

high level of AUC $(>0.8)$ in the ROC. The data were expressed as the odds ratios (ORs) with $95 \%$ confidence intervals (CIs). A P-value of less than 0.05 was considered statistically significant. All analyses were conducted using the SPSS software program, version 25.0. (IBM Corp).

\section{Results}

The characteristics of the patients are summarized in Table I. The median age of the patients was 72 years old (range 37-84), and 43.3 and $56.7 \%$ of the patients were male and female, respectively. Primary diseases were pancreatic cancer in 10 patients (33.3\%), bile duct cancer (33.3\%) in 10, duodenal cancer (23.3\%) in 7 and benign duodenal tumor in $3(10 \%)$. Regarding CR-POPF (grade B and C), grade B POPF occurred in 15 patients (50\%), and grade C POPF was not found in this series. Among the 15 patients with non-CR-POPF, 10 (33.3\%) did not show increased amylase in the drainage fluid, while the remaining 5 (16.7\%) exhibited evidence of BL.

Table II shows the results of the univariate analyses of the patient-related factors with CR-POPF. Patients with non-pancreatic cancers, a smaller pancreatic duct and soft pancreas texture experienced CR-POPF significantly more frequently than others $(\mathrm{P}=0.005, \mathrm{P}=0.004$ and $\mathrm{P}=0.014$, respectively). Among the laboratory data obtained on POD1, a higher white blood cell count, higher levels of serum amylase and serum presepsin and higher concentration of presepsin in the drainage fluid (Fig. 1A) were significantly more common in patients with CR-POPF than in those without CR-POPF $(\mathrm{P}=0.040, \mathrm{P}=0.002, \mathrm{P}=0.012$ and $\mathrm{P}<0.001$, respectively). The postoperative hospital stay was also significantly correlated with CR-POPF ( $\mathrm{P}=0.004)$.

In addition, the white blood cell count, levels of serum amylase and serum presepsin and concentration of presepsin in the drainage fluid on POD1 were examined using ROC curves analyses to predict POPF. The cut-off values of $11,200 / \mu 1$ for the white blood cell count, $399 \mathrm{IU} / \mathrm{l}$ for the serum amylase level, $250 \mathrm{pg} / \mathrm{ml}$ for the serum presepsin level and $1,048.5 \mathrm{pg} / \mathrm{ml}$ for the concentration of presepsin in the drainage fluid were determined using the AUCs of 0.720, 0.836, 0.769 and 0.880, respectively (Table III, Fig. 1B). Furthermore, the serum amylase level and presepsin concentration in the drainage 
Table III. Receiver operating characteristic analysis of variables.

\begin{tabular}{lccccr}
\hline Variables & Cut-off value & AUC & Sensitivity & Specificity & P-value \\
\hline White blood cells on POD1, $\mu 1$ & 11,200 & 0.720 & 0.600 & 0.933 & 0.040 \\
Serum amylase on POD1, IU/1 & 399 & 0.836 & 0.800 & 0.800 & 0.002 \\
Serum presepsin on POD1, pg/ml & 250 & 0.769 & 0.733 & 0.667 & 0.012 \\
Presepsin in drainage fluid on POD1, pg/ml & $1,048.5$ & 0.880 & 0.800 & 0.867 & $<0.001$ \\
\hline
\end{tabular}

AUC, area under the curve; POD1, post-operative day 1.

fluid on POD1 were evaluated using a multivariate analysis to test whether or not they were independent factors predicting CR-POPF. The multivariate logistic regression analysis revealed that a higher level of presepsin in the drainage fluid on POD1 was an independent predictive marker for CR-POPF (OR: 14.503, 95\% CI: 1.750-120.229, $\mathrm{P}=0.013$; Table IV).

The presepsin concentration in the drainage fluid on POD3 was also significantly increased after revealing CR-POPF $(\mathrm{P}=0.001$; Fig. $1 \mathrm{C})$. The ROC analysis with a cut-off level of $1,064.5 \mathrm{pg} / \mathrm{ml}$ demonstrated $90 \%$ accuracy, $80 \%$ sensitivity and $100 \%$ specificity (Fig. 1D). The combined results of presepsin in the drainage fluid in POD1 and POD3 accurately confirmed a diagnosis of CR-POPF (Table V; accuracy: 90\%, sensitivity: $93.3 \%$, specificity: $86.7 \%$ ).

\section{Discussion}

In patients receiving $\mathrm{PD}$, a number of perioperative risk factors of POPF have been reported, including male gender, high body mass index, intra-operative bleeding, soft pancreatic texture, increased pancreatic fat, increased pancreatic parenchymal remnant volume and small pancreatic duct diameter $(1,2,4,7,17,18)$. Many predictive markers, such as postoperative elevated serum amylase, C-reactive protein, serum lipase and lipase in the drainage fluid, have also been developed $(5-7,9,10,19)$. However, the utility of these risk factors and predictive markers of CR-POPF remains controversial. In this study, we confirmed the utility of a novel predictive marker for CR-POPF after PD by focusing on presepsin as a bacterial infection and/or inflammation marker. We demonstrated for the first time that the concentration of presepsin in the drainage fluid was an accurate predictive maker for the detection of CR-POPF.

A recent study suggested that CR-POPF is consistent with $\mathrm{PF}$ accompanied by bacterial infection $(8,14)$. Pancreatic juice contains alkaline digestive enzyme and a high concentration of bicarbonate ions. Enzymatic proteins constitute a large component of the pancreatic juice; for example, trypsin is a crucial digestive enzyme that activates other digestive enzymes $(14,20)$. PF develops after PD due to autolysis caused by activated trypsin, leading to tissue damage. As a result, PF induces intra-abdominal abscess and intra-peritoneal bleeding due to vascular rupture (14). Yamashita et al reported that infection with bacteria involves the secretion of a protease activator of trypsinogen to trypsin. Therefore, controlling bacterial infection in the perioperative period of PD may be crucial for preventing the development of CR-POPF (14). Based on this rationale, we hypothesized that novel bacterial specific inflammatory
Table IV. Multivariate analysis.

\begin{tabular}{lrcr}
\hline & \multicolumn{3}{c}{ Multivariate analysis } \\
\cline { 2 - 4 } Parameters & OR & $(95 \% \mathrm{CI})$ & P-value \\
\hline $\begin{array}{l}\text { Serum amylase on POD1 } \\
\text { Presepsin in drainage fluid } \\
\text { on POD1 }\end{array}$ & $\begin{array}{l}7.817 \\
(0.503\end{array}$ & $(1.750-120.229)$ & 0.013 \\
\end{tabular}

OR, odds ratio; CI, confidence interval; POD, post-operative day.

Table V. Combined results of presepsin in patient drainage fluid on POD1 and POD3.

Clinically relevant postoperative pancreatic fistula

Presepsin in drainage fluid on POD1 and POD3

$(-) n=15 \quad(+) n=15 \quad$ P-value

\begin{tabular}{lrrr}
\hline Both negative & 13 & 1 & $<0.001$ \\
Either positive & 2 & 14 &
\end{tabular}

POD, post-operative day.

markers might be candidates for the earlier and more accurate detection of CR-POPF after PD. In particular, we focused on presepsin, which was identified as a predictive marker for bacterial infection and has been reported to be more sensitive than procalcitonin, a recently identified bacterial-specific inflammatory marker (12). We therefore selected presepsin as a candidate marker for detecting CR-POPF.

We considered that the earlier and more accurate diagnosis of CR-POPF would be closely associated with improving the morbidity and mortality rate, resulting in a better quality of life in patients. The usefulness of serum amylase $(7,9,10)$, C-reactive protein (6), serum lipase (5) and lipase in the drainage fluid (19) as diagnostic markers was previously reported to diagnose CR-POPF as follows: AUC, 0.780-0.793; sensitivity, 81.5-91.7\%; specificity, 55.5-72.7\% for serum amylase (9,10); AUC, 0.796; sensitivity, 94\%; specificity, $62 \%$ for C-reactive protein (6); AUC, 0.76; sensitivity, 92\%; specificity, 66\% for serum lipase (5); AUC, 0.89; sensitivity, 88\%; specificity, $95 \%$ for lipase in drainage fluid (19). However, 
these diagnostic and predictive markers are considered insufficient for the accurate diagnosis of CR-POPF due to their relatively low specificity and sensitivity values. We found that the measurement of presepsin in the drainage fluid on POD1 and 3 was effective for predicting CR-POPF. Furthermore, the combination of the concentrations of presepsin in the drainage fluid on POD1 and POD3 showed even better efficacy for this diagnosis (accuracy: 90.0\%, sensitivity: 93.3\%, specificity: $86.7 \%$ ). In this study, we excluded the amylase concentration in the drainage fluid from the analysis, because the definition of CR-POPF includes the concentration of amylase in the drainage fluid itself. Indeed, the concentration of amylase in the drainage fluid on POD1 was also significantly increased in the CR-POPF group [median: 245 (range 33-3,894) vs. median: 5,009 (range 517-48,620), $\mathrm{P}<0.001$ ] and was highly diagnostic (accuracy: 90\%, sensitivity: 93.3, specificity: 86.7\%). We could prospect that these factors such as presepsin, amylase and/or other candidate factors in the different time point may have an important role to predict the CR-POPF. In addition, multivariate analysis or diagnostic model with combination these factor may improve the prediction of CR-POPF in the future.

The delayed removal of abdominal drains was suggested to be associated with retrograde infection and intra-abdominal complications (21). If true, then optimal drain management is crucial for the early detection of CR-POPF. Conventional ways of detecting infections, such as culture-based approaches, require sophisticated equipment and highly proficient operators and timing. Therefore, we proposed that if the presepsin level in the drain fluid exceeded 1,050 pg/ml (based on our cut-off value of POD1 and POD3 for presepsin in drainage fluid), continuous and additional anti-bacterial therapeutics and/or prolonged drain management may need to be considered.

Several limitations associated with the present study warrant mention. First, the study was conducted at a single institution with a relatively small number of subjects as a pilot study. In addition, the incidence of CR-POPF was relatively high in this study in comparison to the previous study $(2,4-10)$. Therefore, further studies will be needed with a large number of the patients in order to confirm the utility of presepsin as a novel predictive maker for CR-POPF after PD.

In conclusion, the presepsin level in the drainage fluid is useful for detecting CR-POPF after PD.

\section{Acknowledgements}

The authors would like to thank Dr Naoya Kimura, Dr Satomi Nakamura, Dr Hiroaki Nakamura, Dr Kohei Yamada, Dr Keiichiro Okuyama, Dr Osamu Ikeda, Dr Tanaka Toshiya and Dr Seiji Sato (all, Department of Surgery, Saga Medical Center Koseikan) for collecting samples and useful discussions.

\section{Funding}

The current study was supported by Koseikan Institutional Research Grant (grant no. 17-01-01-04).

\section{Availability of data and materials}

All data generated or analyzed during this study are included in this published article.

\section{Authors' contributions}

$\mathrm{MH}$ and AM primarily designed the current study. $\mathrm{MH}, \mathrm{AM}$ and KK treated patients and collected samples and data. YS and MY measured laboratory data, including presepsin. MH and ES analyzed the data. MH, AM, KK, TM and HN interpreted the results and wrote the manuscript. All authors read and approved the final manuscript.

\section{Ethics approval and consent to participate}

All patients and their families provided informed consent for participation after they were fully informed about the surgical procedures of the current study. The Medical Ethics Committee of Saga Medical Center Koseikan reviewed and approved the present study (permission nos. 17-01-01-04 and 19-05-01-01).

\section{Patient consent for publication}

Not applicable.

\section{Competing interests}

The authors declare that they have no competing interests.

\section{References}

1. Rosso E, Casnedi S, Pessaux P, Oussoultzoglou E, Panaro F, Mahfud M, Jaeck D and Bachellier P: The role of 'fatty pancreas' and of BMI in the occurrence of pancreatic fistula after pancreaticoduodenectomy. J Gastrointest Surg 13: 1845-1851, 2009.

2. Kawai M, Kondo S, Yamaue H, Wada K, Sano K, Motoi F, Unno M, Satoi S, Kwon AH, Hatori T, et al: Predictive risk factors for clinically relevant pancreatic fistula analyzed in 1,239 patients with pancreaticoduodenectomy: Multicenter data collection as a project study of pancreatic surgery by the Japanese Society of Hepato-Biliary-Pancreatic Surgery. J Hepatobiliary Pancreat Sci 18: 601-608, 2011

3. Kastenberg ZJ, Morton JM, Visser BC, Norton JA and Poultsides GA: Hospital readmission after a pancreaticoduodenectomy: An emerging quality metric? HPB (Oxford) 15: 142-148, 2013.

4. Kanda M, Fujii T, Suenaga M, Takami H, Hattori M, Inokawa Y, Yamada S, Nakayama G, Sugimoto H, Koike M, et al: Estimated pancreatic parenchymal remnant volume accurately predicts clinically relevant pancreatic fistula after pancreatoduodenectomy. Surgery 156: 601-610, 2014.

5. Dalla Valle R, De Bellis M, Pedrazzi G, Lamecchi L, Bianchi G, Pellegrino $\mathrm{C}$ and Iaria M: Can early serum lipase measurement be routinely implemented to rule out clinically significant pancreatic fistula after pancreaticoduodenectomy? Int J Surg 21 (Suppl 1): S50-S54, 2015.

6. Partelli S, Pecorelli N, Muffatti F, Belfiori G, Crippa S, Piazzai F, Castoldi R, Marmorale C, Balzano G and Falconi M: Early postoperative prediction of clinically relevant pancreatic fistula after pancreaticoduodenectomy: Usefulness of C-reactive Protein. HPB (Oxford) 19: 580-586, 2017.

7. Okabayashi T, Maeda H, Nishimori I, Sugimoto T, Ikeno T and Hanazaki K: Pancreatic fistula formation after pancreaticooduodenectomy; for prevention of this deep surgical site infection after pancreatic surgery. Hepatogastroenterology 56: 519-523, 2009.

8. Nagakawa Y, Matsudo T, Hijikata Y, Kikuchi S, Bunso K, Suzuki Y, Kasuya K and Tsuchida A: Bacterial contamination in ascitic fluid is associated with the development of clinically relevant pancreatic fistula after pancreatoduodenectomy. Pancreas 42: 701-706, 2013

9. Cloyd JM, Kastenberg ZJ, Visser BC, Poultsides GA and Norton JA: Postoperative serum amylase predicts pancreatic fistula formation following pancreaticoduodenectomy. J Gastrointest Surg 18: 348-353, 2014. 
10. Palani Velu LK, Chandrabalan VV, Jabbar S, McMillan DC, McKay CJ, Carter CR, Jamieson NB and Dickson EJ: Serum amylase on the night of surgery predicts clinically significant pancreatic fistula after pancreaticoduodenectomy. HPB (Oxford) 16: 610-619, 2014.

11. Yaegashi Y, Shirakawa K, Sato N, Suzuki Y, Kojika M, Imai S, Takahashi G, Miyata M, Furusako S and Endo S: Evaluation of a newly identified soluble CD14 subtype as a marker for sepsis. J Infect Chemother 11: 234-238, 2005.

12. Chenevier-Gobeaux C, Borderie D, Weiss N, Mallet-Coste T and Claessens YE: Presepsin (sCD14-ST), an innate immune response marker in sepsis. Clin Chim Acta 450: 97-103, 2015.

13. Wright SD, Ramos RA, Tobias PS, Ulevitch RJ and Mathison JC: CD14, a receptor for complexes of lipopolysaccharide (LPS) and LPS binding protein. Science 249: 1431-1433, 1990.

14. Yamashita K, Sasaki T, Itoh R, Kato D, Hatano N, Soejima T, Ishii K, Takenawa T, Hiromatsu K and Yamashita Y: Pancreatic fistulae secondary to trypsinogen activation by Pseudomonas aeruginosa infection after pancreatoduodenectomy. J Hepatobiliary Pancreat Sci 22: 454-462, 2015.

15. Fujii T, Sugimoto H, Yamada S, Kanda M, Suenaga M, Takami H, Hattori M, Inokawa Y, Nomoto S, Fujiwara M and Kodera Y: Modified Blumgart anastomosis for pancreaticojejunostomy: Technical improvement in matched historical control study. J Gastrointest Surg 18: 1108-1115, 2014.
16. Bassi C, Marchegiani G, Dervenis C, Sarr M, Abu Hilal M, Adham M, Allen P, Andersson R, Asbun HJ, Besselink MG, et al: The 2016 update of the International Study Group (ISGPS) definition and grading of postoperative pancreatic fistula: 11 years after. Surgery 161: 584-591, 2017.

17. Del Chiaro M, Rangelova E, Ansorge C, Blomberg J and Segersvard R: Impact of body mass index for patients undergoing pancreaticoduodenectomy. World J Gastrointest Pathophysiol 4: 37-42, 2013.

18. Lee SE, Jang JY, Lim CS, Kang MJ, Kim SH, Kim MA and Kim SW: Measurement of pancreatic fat by magnetic resonance imaging: Predicting the occurrence of pancreatic fistula after pancreatoduodenectomy. Ann Surg 251: 932-936, 2010.

19. Facy O, Chalumeau C, Poussier M, Binquet C, Rat P and Ortega-Deballon P: Diagnosis of postoperative pancreatic fistula. Br J Surg 99: 1072-1075, 2012.

20. Whitcomb DC and Lowe ME: Human pancreatic digestive enzymes. Dig Dis Sci 52: 1-17, 2007.

21. Kawai M, Tani M, Terasawa H, Ina S, Hirono S, Nishioka R, Miyazawa M, Uchiyama K and Yamaue H: Early removal of prophylactic drains reduces the risk of intra-abdominal infections in patients with pancreatic head resection: Prospective study for 104 consecutive patients. Ann Surg 244: $1-7,2006$ 